\title{
Cinética de congelamento do feijão (Phaseolus vulgaris L.) a baixas temperaturas
}

\author{
Mario E. R. M. Cavalcanti-Mata ${ }^{1}$, José O. de Morais ${ }^{1}$, Maria E. M. Duarte ${ }^{1}$, \\ Paulo de A. Farias ${ }^{1}$ \& Alexandre J. de M. Queiroz ${ }^{1}$
}

\begin{abstract}
RESUMO
O objetivo neste trabalho foi obter as curvas de congelamento do feijão, cultivar 'gordo', nas temperaturas de $-25,-50,-170$ e $-196{ }^{\circ} \mathrm{C}$ e determinar sua difusividade térmica efetiva. Com vista à obtenção das curvas de congelamento das sementes nas temperaturas de -25 e $-50{ }^{\circ} \mathrm{C}$ utilizou-se um freezer criogênico horizontal; para a temperatura de $-170{ }^{\circ} \mathrm{C}$, o vapor de nitrogênio e, para a temperatura de $-196{ }^{\circ} \mathrm{C}$, as sementes foram submersas no nitrogênio líquido. Afim de expressar o comportamento cinético do congelamento das sementes de feijão, foi usado o modelo de Fourier levando-se em consideração o primeiro termo da série, além dos Modelos I e II de Cavalcanti-Mata \& Duarte. Os resultados obtidos indicam que os três modelos representam satisfatoriamente os dados experimentais da cinética de congelamento; apesar disto, com o Modelo II de Cavalcanti-Mata \& Duarte se obtém o maior coeficiente de determinação. Constata-se também que, para congelar as sementes de feijão até atingir o equilíbrio térmico na temperatura de $-25{ }^{\circ} \mathrm{C}$, o tempo necessário foi de $1200 \mathrm{~min}$; em temperatura de $-50{ }^{\circ} \mathrm{C} \mathrm{o}$ equilíbrio foi atingido com 480 min; a $-170{ }^{\circ} \mathrm{C}$ o equilíbrio se deu em $180 \mathrm{~min}$ e, a $-196{ }^{\circ} \mathrm{C}$, em 30 min.
\end{abstract}

Palavras-chave: criogenia, sementes, difusividade térmica, energia de ativação

\section{Freezing kinetics of bean (Phaseolus vulgaris L.) at low temperatures}

\begin{abstract}
The objective of this study was to obtain the freezing curves of beans, variety 'gordo' at temperatures of $-25,-50,-170$ and $-196{ }^{\circ} \mathrm{C}$, and determine their effective thermal diffusivity. For the curves of freezing beans seeds at temperatures of -25 and $-50{ }^{\circ} \mathrm{C}$ a cryogenic horizontal freezer was used, for temperature of $-170{ }^{\circ} \mathrm{C}$, nitrogen vapor was used and at temperature of $-196{ }^{\circ} \mathrm{C}$ seeds were submerged in liquid nitrogen. To express the kinetic behavior of the freezing of bean seeds, the Fourier model was used taking into account the first term of the series and; Cavalcanti-Mata \& Duarte model I and II. The results indicate that the three models satisfactorily represent the experimental data of the kinetics of freezing, though with Model II of Cavalcanti-Mata \& Duarte highest coefficient of determination were obtained. It is concluded that time necessary to freeze the seeds of beans at temperature of $-25^{\circ} \mathrm{C}$ was of $1200 \mathrm{~min}$; at temperature of $-50{ }^{\circ} \mathrm{C}$ the equilibrium was reached with $480 \mathrm{~min}$; at $-170{ }^{\circ} \mathrm{C}$ the time was $180 \mathrm{~min}$ and at $-196{ }^{\circ} \mathrm{C}$ it was $30 \mathrm{~min}$.
\end{abstract}

Key words: cryogenics, seeds, thermal diffusivity, activation energy 


\section{INTRODUÇÃO}

O feijão (Phaseolus vulgaris L.) é consumido no Brasil, principalmente no nordeste (Silva et al., 2008). Este alimentoé um dos mais tradicionais na dieta do brasileiro,pois fornece nutrientes essenciais ao ser humano, como proteínas, ferro, cálcio, magnésio, zinco, carboidratos, fibras e vitaminas, sobretudo do complexo B. O feijão ocupa o terceiro lugar entre os alimentos consumidos, totalizando $11,2 \%$ das calorias ingerida; trata-se, portanto, da principal fonte de proteínas e calorias das populações de baixa renda. $\mathrm{O}$ valor nutritivo da proteína do feijão é baixo quando utilizado como única fonte protéica mas, se combinado com o arroz, forma uma mistura de proteínas mais nutritiva. Junto com a carne bovina esses alimentos básicos correspondem a $70 \%$ da ingestão protéica, tornando o feijão interno uma cultura de grande expressão socioeconômica, razão por que constantes variedades vêm sendo desenvolvidas no país nas últimas década, visando obter variedades mais produtivas e mais resistentes a doenças. Este fato pode ser observado em dados estatísticos do IBGE (2011) através dos quais se verifica que na década de 70 a produtividade do feijão era de $500 \mathrm{~kg} \mathrm{ha}^{-1}$, nos anos 90 a produtividade passou para $750 \mathrm{~kg} \mathrm{ha}^{-1} \mathrm{e}$, na década dos anos 2000, foi de $900 \mathrm{~kg} \mathrm{ha}^{-1}$; contudo, ainda está distante da produtividade mexicana ou americana, de $1400 \mathrm{~kg} \mathrm{ha}^{-1}$.

Para que as constantes pesquisas continuem sendo realizadas há necessidade de se preservar as atuais cultivares em bancos de germoplasma. No banco de germoplasma as sementes de determinada espécie são armazenadas com objetivo de fornecer matéria-prima para a engenharia genética ou para os programas de melhoramento vegetal, com a finalidade de criar novas cultivares mais produtivas para diversas condições edafoclimáticas e mais resistentes a pragas e doenças (Almeida et al., 2010; Tresena et al., 2009). Um dos processos indispensáveis à preservação essas espécies no banco de germoplasma é o congelamento do material genético que pode variar da temperatura de $-20{ }^{\circ} \mathrm{C}$ até temperaturas criogênicas $\left(-196^{\circ} \mathrm{C}\right)$. De acordo com Cavalcanti-Mata \& Duarte (2011) o congelamento a baixas temperaturas pode ser dividido em 3 nomenclaturas: congelamento convencional (lento), em que o processo ocorre em temperaturas que vão de 0 a $-60{ }^{\circ} \mathrm{C}$; congelamento semicriogênico (rápido), cujo processo ocorre nas temperaturas entre $-60 \mathrm{a}-130^{\circ} \mathrm{C}$ e congelamento criogênico (ultra-rápido) em que o processo ocorre entre -130 a -196 ${ }^{\circ} \mathrm{C}$.

Segundo Cavalcanti-Mata (2008) existem sementes que não toleram o congelamento uma vez que perdem sua viabilidade durante este processo; no entanto, outras sementes toleram o congelamento e são ditas sementes passíveis de serem conservadas a baixas temperaturas.

Para as sementes que têm esta particularidade, torna-se imprescindível conhecer as características da cinética de congelamento que possibilitam determinar os tempos necessários para que as sementes possam ser congeladas em diferentes temperaturas podendo-se determinar sua difusividade térmica efetiva, que é a propriedade física deste produto biológico que estabelece como a energia se propaga da parte externa até o interior do produto (Becker \& Fricke, 1999; Fikiin \& Fikiin, 1999; Bonomo et al., 2009; Barbin et al., 2010).
Assim, o objetivo nesta pesquisa foi estudar a cinética de congelamento do feijão, cultivar 'gordo', nas temperaturas de $-25,-50,-170$ e $-196^{\circ} \mathrm{C}$, determinando sua difusividade térmica efetiva para referido intervalo de temperatura e como calcular a energia de ativação.

\section{MATERIAL E MÉTODOS}

O trabalho foi realizado no setor de criogenia do Laboratório de Armazenamento e Processamento de Produtos Agrícolas da Unidade Acadêmica de Engenharia Agrícola da Universidade Federal de Campina Grande. As sementes, adquiridas do banco de sementes de produtores locais da cidade de Campina Grande, foram selecionadas com a melhor aparência externa, casca íntegra, sem furos, rachaduras ou manchas, causadas por agentes externos. Inicialmente, 100 sementes foram selecionadas e determinadas suas dimensões de comprimento, largura e espessura, com auxílio de um paquímetro digital, marca Mitutuyo com resolução de 0,01 mm.

Determinou-se o volume da semente de feijão considerandose o feijão um elipsóide (Eq. 1); posteriormente, utilizou-se o procedimento descrito por Cavalcanti-Mata \& Duarte (2011) sugerido por Mohsenin (1992) para equacionamento de modelos matemáticos conhecendo-se, daí, o volume do material determinando-se, então, o raio da esfera e se considerando este formato para o feijão. Referido dado foi utilizado no modelo matemático de Fourier e no Modelo I, proposto por CavalcantiMata \& Duarte.

$$
\mathrm{V}=\frac{4}{3} \pi\left(\begin{array}{lll}
\mathrm{r}_{1} & \mathrm{r}_{2} & \mathrm{r}_{3}
\end{array}\right)
$$

em que:

$$
\begin{array}{ll}
r_{1} & \text { - raio maior do feijão } \\
r_{2} & \text { - raio intermidiário } \\
r_{3} & \text { - raio menor }
\end{array}
$$

Visando determinar a curva de congelamento introduziu-se, no centro geométrico da semente de feijão (teor de água de $10,8 \%$ base úmida) um termopar de $0,2 \mathrm{~mm}$ de diâmetro acoplado a um registrador Digi-Sensi de dois canais; o outro termopar foi introduzido no interior da unidade de congelamento cuja finalidade foi monitorar o meio congelante, de modo a se determinar o instante de equilíbrio térmico para cada temperatura de congelamento.

Para o estudo da cinética de congelamento da semente de feijão cultivar 'gordo' na temperatura de $-25 \pm 2{ }^{\circ} \mathrm{C}$, foi utilizado um freezer horizontal e os dados coletados a cada $30 \mathrm{~s}$; para a temperatura de $-50 \pm 2{ }^{\circ} \mathrm{C}$ utilizou-se um balcão criogênico horizontal e os dados foram obtidos a cada $10 \mathrm{~s}$; para estudo da cinética de congelamento das sementes na temperatura de $170{ }^{\circ} \mathrm{C}$, utilizou-se um depósito criogênico cujo vapor do nitrogênio forneceu a temperatura de $-170{ }^{\circ} \mathrm{C}$ e os dados registrados a cada $5 \mathrm{~s}$, conforme ilustrado na Figura 1A. Para se determinar a cinética de congelamento na temperatura de - 
A.

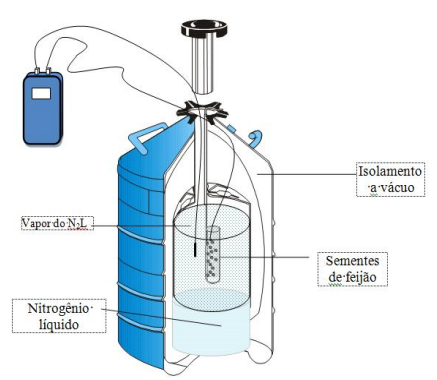

B.

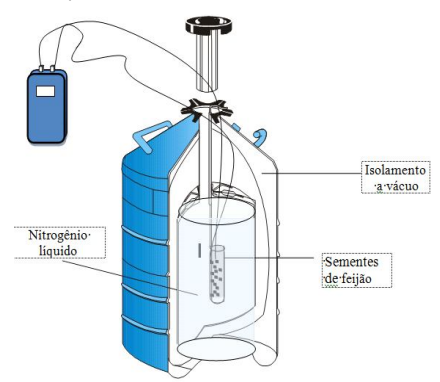

Figura 1. Depósito para congelamento da semente de feijão nas temperaturas de -170 (A) e $-196^{\circ} \mathrm{C}$ (B)

$196{ }^{\circ} \mathrm{C}$, as sementes foram imersas no nitrogênio líquido em um depósito criogênico (Figura 1B) e os dados foram obtidos a cada 2 s. O término dos registros das temperaturas aconteceu quando o segundo termopar atingia a temperatura de congelamento em cada câmara. Todos os dados deste experimento para obtenção da cinética do congelamento foram feitos com 10 repetições.

\section{Tratamento matemático}

Os modelos matemáticos utilizados para descrever a cinética de congelamento nas diversas temperatura (-25, -50, -170 e 196 ${ }^{\circ} \mathrm{C}$ ) foram os de Fourier, utilizando o primeiro termo da série, o Modelo I e Modelo II, propostos por Cavalcanti-Mata \& Duarte (2011).

O Modelo de Fourier utilizando-se o $1^{\circ}$ termo da série, deriva da equação geral de transferência de calor (Eq. 2).

$$
\frac{\partial T}{\partial t}=\frac{\alpha}{r^{2}}\left[\frac{\partial}{\partial r}\left(r^{2} \frac{\partial T}{\partial r}\right)\right]
$$

A solução analítica da equação foi dada por Apaci, de acordo com Mohsenin (1992) quando a temperatura foi obtida no centro da esfera.

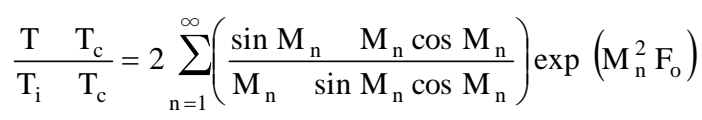

em que:

$$
\begin{gathered}
\frac{T-T_{c}}{T_{i}-T_{c}}=R T \\
\frac{2}{M_{1}}\left(\frac{\sin M_{1} \quad M_{1} \cos M_{1}}{M_{1} \sin M_{1} \cos M_{1}}\right)=J \\
F_{o}=\frac{\alpha t}{r_{m}^{2}}
\end{gathered}
$$

Substituindo-se as Eqs. 3, 4 e 5 em , tem-se:

$$
\mathrm{RT}=\mathrm{J} \exp \left(\frac{\mathrm{M}_{1}^{2} \alpha \mathrm{t}}{\mathrm{r}_{\mathrm{m}}^{2}}\right)
$$

em que:

$$
\mathrm{k}=\left(\frac{\mathrm{M}_{1}^{2} \alpha}{\mathrm{r}_{\mathrm{m}}^{2}}\right)
$$

em que:

RT - razão de temperatura, adimensional

$\mathrm{T}$ - temperatura do produto no tempo $\mathrm{t},{ }^{\circ} \mathrm{C}$

$\mathrm{T}_{c}$ - temperatura do meio de congelamento, ${ }^{\circ} \mathrm{C}$

$\mathrm{T}_{\mathrm{i}}^{\mathrm{c}}$ - temperatura inicial do produto, ${ }^{\circ} \mathrm{C}$

$\mathrm{F}_{\mathrm{o}}$ - número de Fourier, adimensional

J - fator de atraso

$\mathrm{k}$ - constante de resfriamento

$\mathrm{M}_{\mathrm{n}}$ - raiz transcendental

$\alpha$ - difusividade térmica efetiva, $\mathrm{mm}^{2} \mathrm{~min}^{-1}$

$r_{m}$ - raio da semente de feijão, $\mathrm{mm}$

$\mathrm{t}$ - tempo, min

O Modelo I de Cavalcanti-Mata \& Duarte (2011) propõe uma equação derivada do Modelo de Fourier, utilizando-se o $1^{\circ}$ termo da série. Neste modelo os autores ressaltam haver uma correção exponencial $(\mathrm{N})$ no tempo e seu modelo pode ser escrito como:

$$
\mathrm{RT}=\mathrm{J}^{\prime} \exp \left(\frac{\mathrm{M}_{1}^{2} \alpha}{\mathrm{r}_{\mathrm{m}}^{2}}\right) \mathrm{t}^{\mathrm{N}}
$$

em que:

J' - fator de atraso, variando ente 1,01 e 1,04

O Modelo II proposto por Cavalcanti-Mata \& Duarte (2011) é um modelo empírico, em que os autores introduzem dois coeficientes na cinética de congelamento enunciando que no comportamento cinético existe uma mudança de curvatura e, desta forma, o modelo pode ser escrito como:

$$
\mathrm{RT}=\mathrm{A} \exp \left(\frac{\psi_{1}}{\mathrm{r}_{\mathrm{m}}^{2}}\right) \mathrm{t}^{\mathrm{N}_{1}}+\mathrm{B} \exp \left(\frac{\psi_{2}}{\mathrm{r}_{\mathrm{m}}^{2}}\right) \mathrm{t}^{\mathrm{N}_{2}}
$$

em que:

$\psi_{1}$ e $\psi_{2}$ - constantes da cinética de congelamento

A, B, $\mathrm{N}_{1}$ e $\mathrm{N}_{2}$ - coeficientes da equação de congelamento

Para determinação da difusividade térmica efetiva a partir das Eqs. 6 e 8, foram obtidos os coeficientes das equações J , J' e A, por meio do programa computacional Statistic 7.0.

Obteve-se a energia de ativação $\left(E_{a}\right)$ a partir da dependência da difusividade térmica efetiva $(\alpha)$ com a temperatura analisada pela equação de Arrhenius (Horn et al., 2010) : 


$$
\alpha=D_{\mathrm{o}} \cdot \exp \left(\frac{\mathrm{E}_{\mathrm{a}}}{\mathrm{R}(\mathrm{T})}\right)
$$

em que:

$$
\begin{aligned}
& D_{o} \text { - constante, } \mathrm{m}^{2} \mathrm{~s}^{-1} \\
& \mathrm{E}_{\mathrm{a}} \text { - energia de ativação, } \mathrm{J} \mathrm{mol}^{-1} \\
& \mathrm{R} \text { - constante universal dos gases, } 8,314 \mathrm{~J} \mathrm{~mol}^{-1} \mathrm{~K}^{-1} \\
& \mathrm{~T}
\end{aligned}
$$

Para o ajuste dos modelos foram realizadas análises de regressão não linear, pelo método Gauss-Newton. Para cada modelo foram considerados o coeficiente de determinação $\left(\mathrm{R}^{2}\right)$, o erro médio estimado (SE) e o erro médio relativo $(\mathrm{P})$.

$$
\begin{gathered}
\mathrm{SE}=\sqrt{\frac{\sum\left(\mathrm{RT}_{\exp _{\mathrm{i}}} \mathrm{RT}_{\mathrm{pre}_{\mathrm{i}}}\right)^{2}}{\mathrm{GLR}}} \\
\mathrm{P}=\frac{100}{\mathrm{n}} \sum_{\mathrm{i}=1}^{\mathrm{n}} \frac{\mid\left(\mathrm{RT}_{\exp _{\mathrm{i}}} \mathrm{RT}_{\mathrm{pre}_{\mathrm{i}}}\right)}{\mathrm{RT}_{\exp _{\mathrm{i}}}}
\end{gathered}
$$

em que:

$\mathrm{RT}_{\text {pre }_{i}}$ - razão de temperatura predita pelo modelo

$\mathrm{RT}_{\text {exp }}$ - razão de temperatura experimental

n - número de observações do experimento

GLR - grau de liberdade do modelo.

\section{RESULTADOS E DISCUSSÃO}

O comportamento cinético do congelamento das sementes de feijão, cultivar 'gordo', nas temperaturas de -25, -50, -170 e $-196{ }^{\circ} \mathrm{C}$ encontra-se nas Figuras $2 \mathrm{~A}, \mathrm{~B}$ e C, respectivamente, para os Modelos de Fourier, Modelo I de Cavalcanti-Mata \& Duarte e Modelo II de Cavalcanti-Mata \& Duarte. Observase que o Modelo II, de Cavalcanti-Mata \& Duarte, apresenta um ajuste melhor entre os dados calculados e os experimentais, destacando-se que este modelo é empírico e não permite determinar a difusividade térmica do processo de congelamento da semente de feijão e, sim, uma constante de congelamento (L). Os outros dois modelos que permitem obter a difusividade térmica efetiva do processo de congelamento do feijão têm um bom ajuste entre os dados experimentais e os estimados em toda a extensão da curva, apenas para a temperatura de $-50{ }^{\circ} \mathrm{C}$, indica que o Modelo de Fourier utilizando apenas o primeiro termo da série, não permite expressar a cinética de congelamento para a maioria das temperaturas estudadas, sinalizando que um número maior da série deve ser utilizado.

Na Tabela 1 se encontram os parâmetros do feijão 'gordo' $\left(\mathrm{r}_{\mathrm{m}}, \mathrm{r}_{\mathrm{m}}^{2}\right)$, coeficientes dos modelos $\left(\mathrm{J}, \mathrm{J}\right.$ ', $\left.\mathrm{M}_{1}\right)$, tal como os coeficientes de determinação das curvas de congelamento do feijão, seu erro médio estimado e o erro médio relativo, além de sua difusividade térmica efetiva. Observa-se que a difusividade
A.

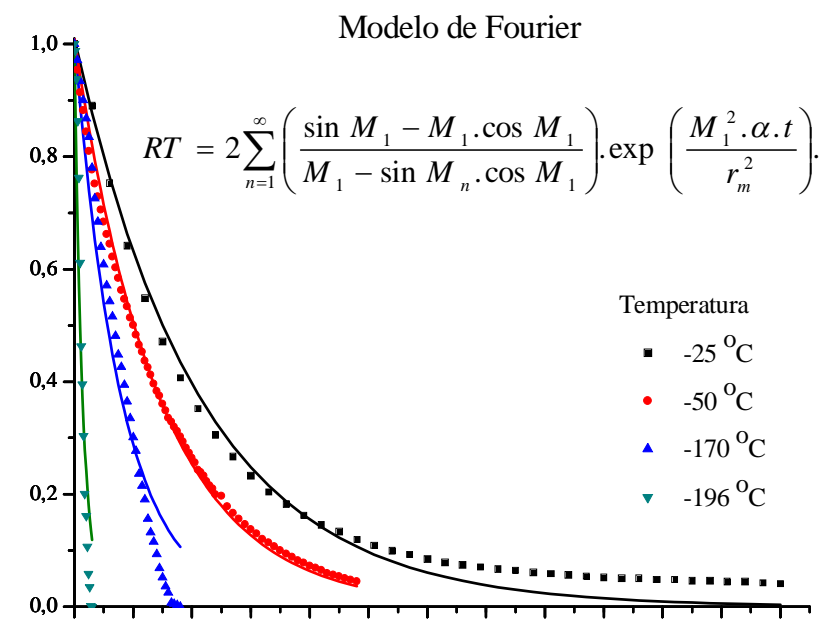

B.

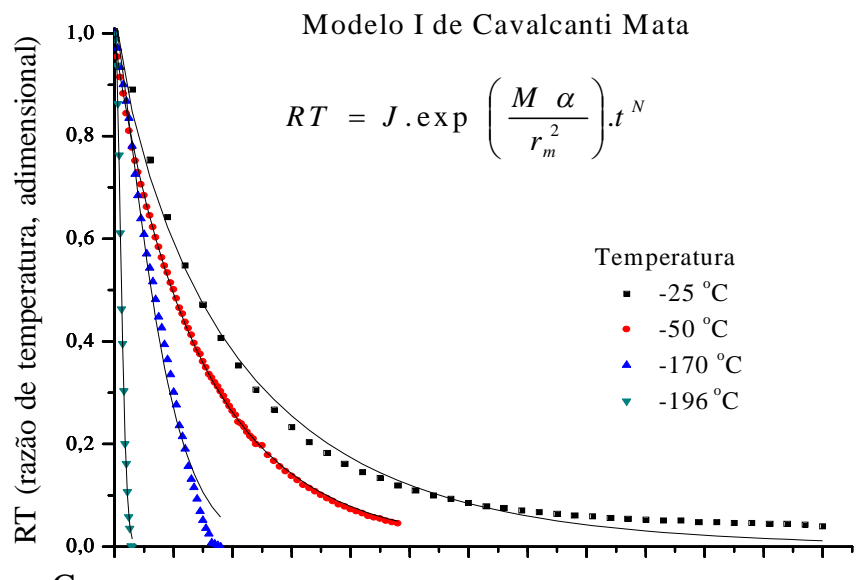

C.

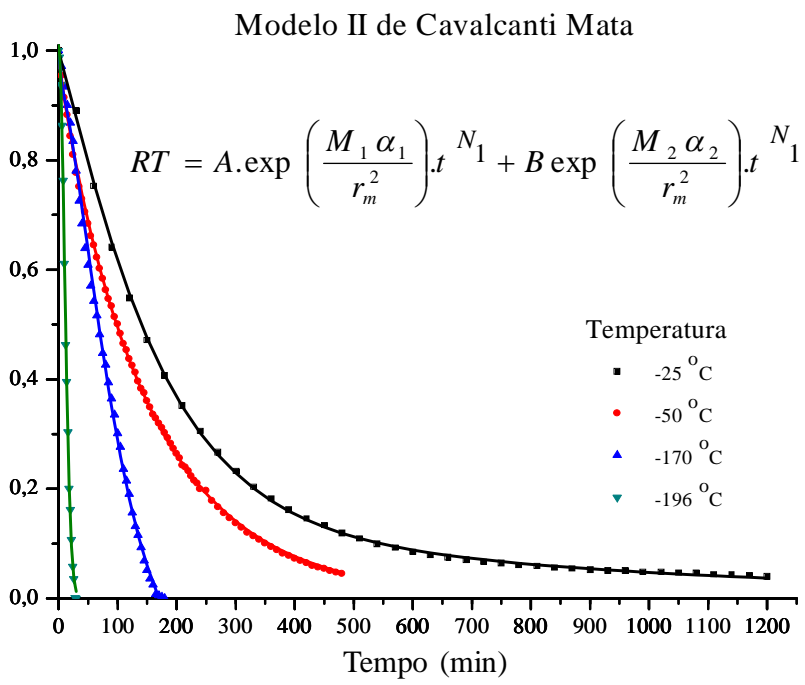

Figura 2. Dados experimentais e calculados pelo Modelo de Fourier com o $1^{\circ}$ termo da série (A), pelo Modelo I de Cavalcanti-Mata \& Duarte (B) e pelo Modelo II de Cavalcanti-Mata \& Duarte (C), da curva de congelamento de sementes de feijão cultivar 'gordo' nas temperaturas de $-25,-50,-170$ e $-196{ }^{\circ} \mathrm{C}$

térmica efetiva do feijão aumenta com a diminuição da temperatura, de -25 para $-196{ }^{\circ} \mathrm{C}$ para o Modelo de Fourier e o Modelo I de Cavalcanti-Mata \& Duarte (2011). 
Tabela 1. Parâmetros da semente de feijão, cultivar 'gordo' e coeficientes dos modelos de cinética de congelamento dessa semente

\begin{tabular}{|c|c|c|c|c|c|c|c|c|c|c|}
\hline $\begin{array}{l}\text { Temperatura } \\
\left({ }^{\circ} \mathrm{C}\right)\end{array}$ & J & $\left(\frac{\mathbf{M}_{1}^{2} \alpha}{\mathbf{r}_{m}^{2}}\right)$ & $\underset{(\mathrm{mm})}{\mathbf{r}_{\mathrm{m}}}$ & $\underset{\left(\mathrm{mm}^{2}\right)}{\mathbf{r}_{\mathbf{m}}^{2}}$ & $M_{1}$ & $\begin{array}{c}\alpha \\
\left(\mathrm{mm}^{2} \min ^{-1}\right)\end{array}$ & & $\begin{array}{l}R^{2} \\
(\%)\end{array}$ & SE & $\begin{array}{c}P \\
(\%)\end{array}$ \\
\hline \multicolumn{11}{|c|}{ Modelo de Fourier ( $1^{0}$ termo da série) } \\
\hline-25 & 1,01 & $-0,00468$ & 4,26 & 18,15 & 0,316 & 0,2688 & & 98.6 & 0,0286 & 37,8 \\
\hline-50 & 1,01 & $-0,00691$ & 4,27 & 18,23 & 0,316 & 0,3987 & & 99,6 & 0,0164 & 5,7 \\
\hline-170 & 1,01 & $-0,01252$ & 4,32 & 18,66 & 0,316 & 0,7394 & & 94.8 & 0,0723 & 31,1 \\
\hline-196 & 1,01 & $-0,07145$ & 4,23 & 17,89 & 0,316 & 4,0457 & & 89,4 & 0,1188 & 53,6 \\
\hline $\begin{array}{l}\text { Temperatura } \\
\left({ }^{\circ} \mathrm{C}\right)\end{array}$ & $\mathbf{J}$ & $\left(\frac{M_{1}^{2} \alpha}{r_{m}^{2}}\right)$ & N & $\underset{\left(\mathrm{mm}^{2}\right)}{\mathbf{r}_{\mathbf{m}}^{2}}$ & $M_{1}$ & $\begin{array}{c}\alpha \\
\left(\mathrm{mm}^{2} \min ^{-1}\right)\end{array}$ & & $\begin{array}{l}R^{2} \\
(\%)\end{array}$ & SE & $\begin{array}{c}P \\
(\%)\end{array}$ \\
\hline \multicolumn{11}{|c|}{ Modelo I de Cavalcanti-Mata \& Duarte (2011) } \\
\hline-25 & 1,04 & $-0,01193$ & 0,83634 & 18,15 & 0,627 & 0,3453 & & 99,20 & 0,0211 & 23,3 \\
\hline-50 & 1,02 & $-0,00988$ & 0,92609 & 18,23 & 0,445 & 0,4048 & & 99,62 & 0,0050 & 2,7 \\
\hline-170 & 1,00 & $-0,00119$ & 1,51701 & 18,66 & 0,050 & 0,4442 & & 99.21 & 0,0367 & 22,5 \\
\hline-196 & 1,00 & $-0,00588$ & 1,93405 & 17,89 & 0,050 & 2,1042 & & 99,81 & 0,0163 & 4,5 \\
\hline $\begin{array}{l}\text { Temperatura } \\
\left({ }^{\circ} \mathrm{C}\right)\end{array}$ & k & $\left(\frac{\psi_{1}}{\mathbf{r}_{m}^{2}}\right)$ & $\mathrm{N}_{1}$ & $\mathbf{r}_{m}^{2}$ & B & $\left(\frac{\psi_{2}}{r_{m}^{2}}\right)$ & $\mathrm{N}_{2}$ & $\begin{array}{l}R^{2} \\
(\%)\end{array}$ & SE & $\begin{array}{c}P \\
(\%)\end{array}$ \\
\hline \multicolumn{11}{|c|}{ Modelo II de Cavalcanti-Mata \& Duarte (2011) } \\
\hline-25 & 0,76866 & $-0,00267$ & 1,15997 & 18,2 & 0,230677 & $-0,00731$ & 0,779 & 99,97 & 0,0040 & 3,2 \\
\hline-50 & 0,14166 & $-0,01391$ & 0,87988 & 18,2 & 0,845378 & $-0,00828$ & 0,955 & 99,97 & 0,0045 & 1,9 \\
\hline-170 & $-0,02220$ & 0,91253 & 0,12406 & 18,2 & 1,018261 & $-0,8910^{-3}$ & 1,509 & 99,77 & 0,0152 & 9,5 \\
\hline-196 & 0,52260 & $-0,00481$ & 2,03703 & 18,2 & 0,486717 & $-0,00431$ & 2,008 & 99,82 & 0,0153 & 3,6 \\
\hline
\end{tabular}

Analisando os dois modelos verifica-se que a difusividade térmica aparente nas temperaturas de -170 e $-196^{\circ} \mathrm{C}$, para o Modelo de Fourier, é praticamente o dobro da estimada pelo Modelo de Cavalcanti-Mata \& Duarte. Este valor de difusividade térmica aparente se mantém na mesma ordem de grandeza das sementes de pinhão-manso congelado na temperatura de $-170{ }^{\circ} \mathrm{C}$ e obtido por Goldfarb et al. (2010), de $0,126 \mathrm{~mm}^{2} \mathrm{~min}^{-1}$; no entanto, para o congelamento do pinhãomanso, feito a $-196^{\circ} \mathrm{C}$, a difusividade foi de $0,19548 \mathrm{~mm}^{2} \mathrm{~min}^{-1}$ que está na ordem de grandeza dez vezes menor que a obtida para o feijão, fato que pode ser explicado pela constituição porosa das sementes de pinhão- manso, que funciona como isolante térmico e também pelo elevado nível de óleo do pinhãomanso (50\%), em cujo comportamento a temperatura se difunde lentamente.

Para a temperatura de $-50{ }^{\circ} \mathrm{C}$ os valores da difusividade térmica efetiva são semelhantes haja vista que no Modelo de Fourier foi de $0,3987 \mathrm{~mm}^{2} \mathrm{~min}^{-1} \mathrm{e}$, pelo Modelo I, foi de 0,4048 $\mathrm{mm}^{2} \mathrm{~min}^{-1}$; apesar disto, para a temperatura de $-25^{\circ} \mathrm{C}$ no modelo I de Cavalcanti-Mata \& Duarte $\left(0,3457 \mathrm{~mm}^{2} \mathrm{~min}^{-1}\right)$ a difusividade térmica efetiva foi maior que pelo Modelo de Fourier $(0,2688$ $\mathrm{mm}^{2} \mathrm{~min}^{-1}$ ).

Ao se comparar o comportamento cinético do congelamento do feijão observa-se haver coerência na representação dos modelos uma vez que no congelamento das semente, feito na temperatura de $-50{ }^{\circ} \mathrm{C}$, as curvas dos dados calculados pelo Modelo de Fourier e pelo Modelo I de Cavalcanti-Mata \& Duarte têm coeficientes de determinação semelhantes (99,6 e $99,62 \%$ ) o que faz com que os dados experimentais e os dados estimados representem bem o fenômeno de congelamento do feijão e o valor das difusividades dos dois modelos sejam praticamente iguais; contudo, quando se analisa o comportamento da cinética de congelamento do feijão na temperatura de $-25,-170$ e $-196{ }^{\circ} \mathrm{C}$, o Modelo de Fourier tem coeficiente de determinação inferior (98,6; 94,8 e 89,4\%, respectivamente) e erros médios estimados superiores $(0,0286$; 0,$0723 ; 0,1188$, respectivamente), além de erro médio relativo (P) superior a $10 \%$, indicando que o modelo de Fourier utilizando o $1^{\circ}$ termo da série não representa tão bem a cinética de congelamento quanto o Modelo I de Cavalcanti-Mata \& Duarte, em que os coeficientes de determinação são de 99,2, 99,21 e $99,81 \%$, respectivamente para as temperaturas de congelamento de $-25,-170$ e $-196^{\circ} \mathrm{C}$, com erros médios estimados (SE) e erros médios relativos (P) inferiores. Essas observações permitem concluir que os dados de difusividade térmica efetiva obtidos pelo Modelo I de Cavalcanti-Mata \& Duarte são mais consistentes e devem ser considerados quando se fizer necessário utilizá-los nos processos de fenômenos de transporte, embora se constate, na Tabela 1, que nas temperaturas -50 e $-170{ }^{\circ} \mathrm{C}$ o erro médio relativo (P) do Modelo I de Cavalcanti-Mata \& Duarte esteja acima de 10\% (23,3 e $22,5 \%$, respectivamente), valor este não recomendado para a seleção de modelos, de acordo com Mohapatra \& Rao (2005).

Percebe-se também, na comparação entre os modelos, que um modelo empírico como o Modelo II de Cavalcanti-Mata \& Duarte tem sua utilidade, representando melhor os dados experimentais que os outros dois modelos; entretanto, não é possível extrair, desse modelo, a difusividade térmica efetiva mas se pode obter uma constante de congelamento, que é função do raio da semente e da temperatura do produto.

Embora os modelos propostos sejam, de maneira geral, satisfatórios para expressar a cinética de congelamento do feijão, constata-se que os gráficos dos valores dos resíduos das equações versus valores estimados, são todos considerados 
tendenciosos, como se observa nas Figuras 3, 4 e 5, respectivamente, para os modelos de Fourier, I de CavalcantiMata \& Duarte e II de Cavalcanti-Mata \& Duarte, sinalizando os

Modelo de Fourier $\left(1^{\circ}\right.$ termo da série $)$
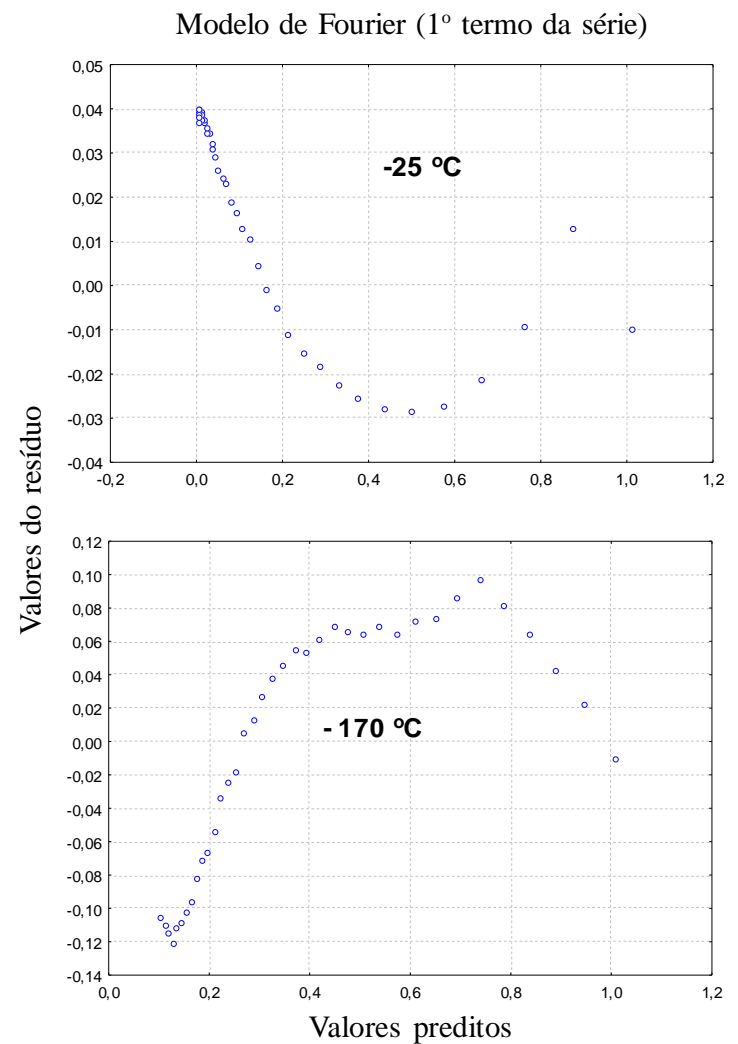

que modelos mais expressivos poderiam ser utilizados; no entanto, há que se desenvolver um modelo que considere o fenômeno fisico que ocorre durante o congelamento das sementes.

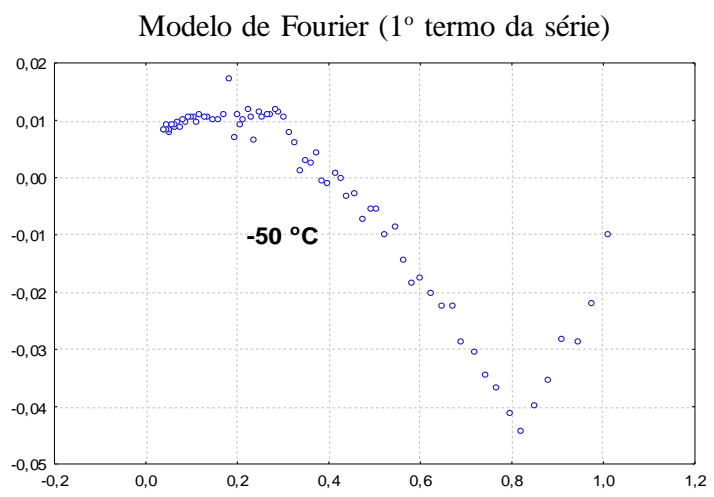

Figura 3. Valores do resíduo versus os valores preditos das curvas de congelamento a $-25,-50,-170$ e $-196{ }^{\circ} \mathrm{C}$ ddo Modelo de Fourier
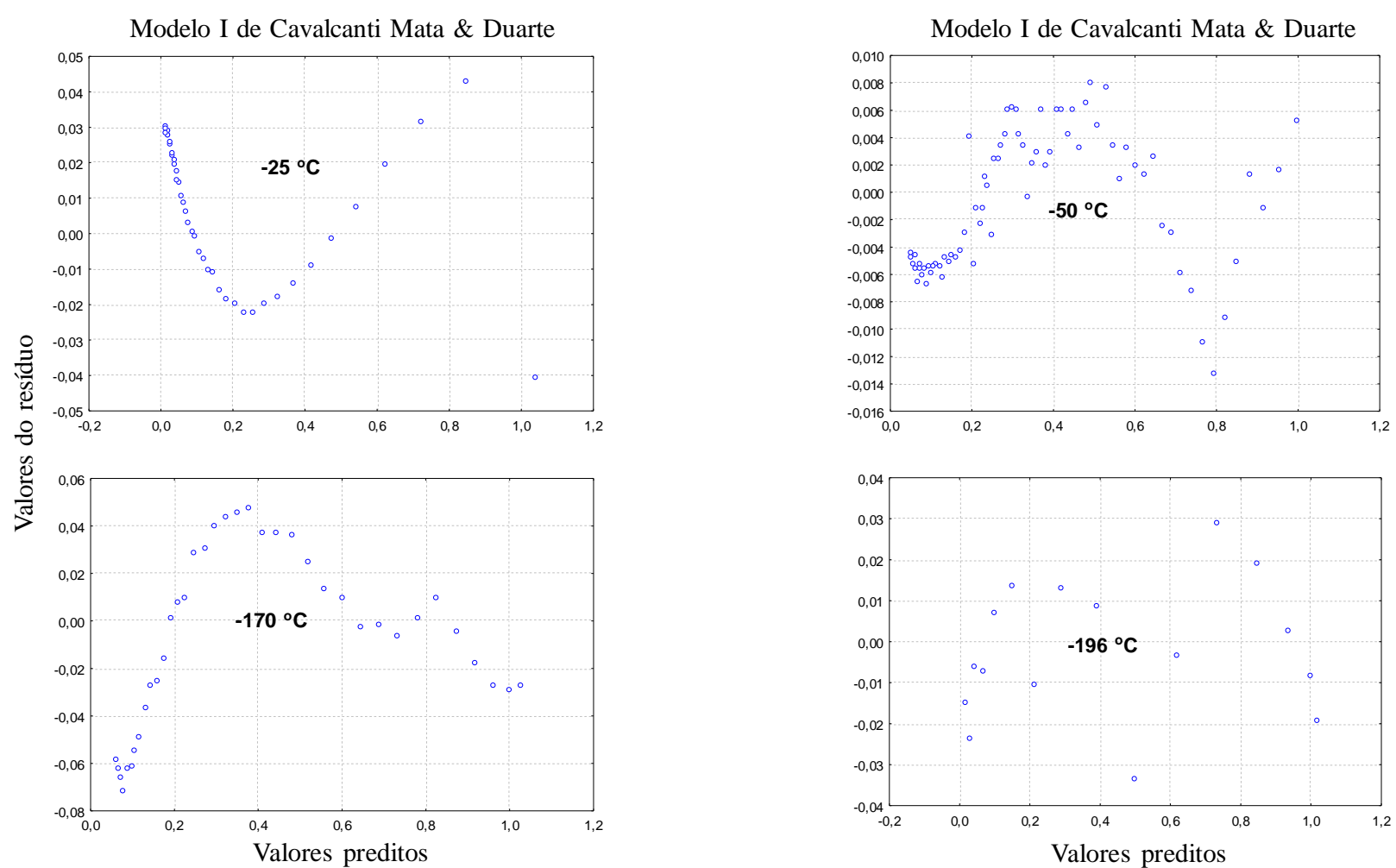

Figura 4. Valores do resíduo versus valores preditos das curvas de congelamento a -25, -50, -170 e $-196^{\circ} \mathrm{C}$ do $\mathrm{Modelo}$ I de Cavalcanti-Mata \& Duarte 

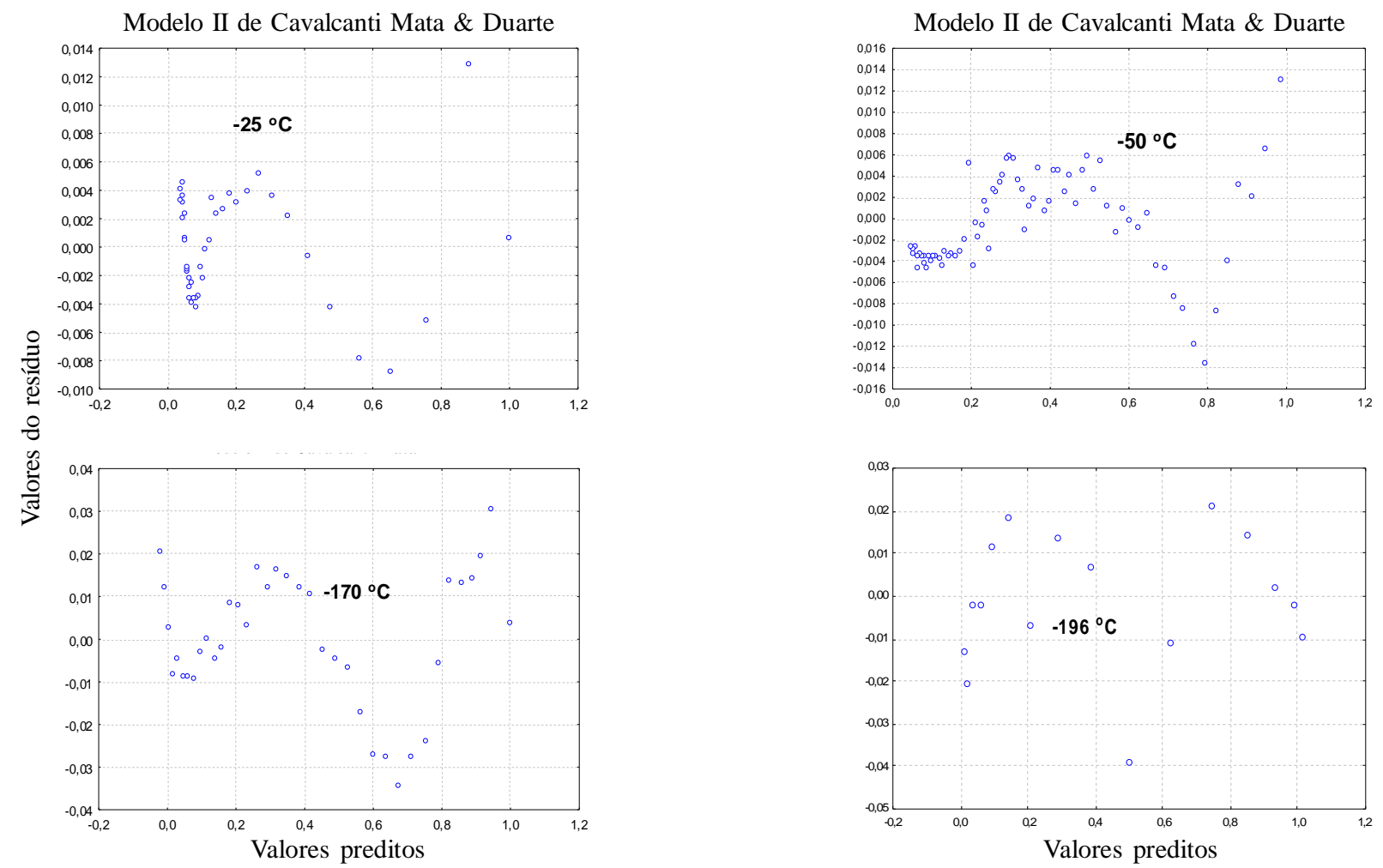

Figura 5. Valores do resíduo versus valores preditos das curvas de congelamento a $-25,-50,-170$ e $-196{ }^{\circ} \mathrm{C}$ do Modelo Il de Cavalcanti-Mata \& Duarte

Na Figura 6 A e B se encontra a relação da difusividade térmica efetiva obtida do Modelo de Fourier e do Modelo I de Cavalcanti-Mata \& Duarte, respectivamente, em função da temperatura em K. A equação utilizada foi a de Arrenius (Eq. 10); esta equação é utilizada para explicar as reações químicas. A ocorrência de uma reação química está relacionada, obrigatoriamente, com o contato entre as moléculas reagentes e a uma energia mínima necessária. Esta energia mínima para a ocorrência da reação é chamada energia de ativação; portanto, pode-se utilizar a equação de Arrenius para determinar a energia de ativação do processo de congelamento, entendendo que esta seria a energia mínima necessária para que ocorram os rearranjos moleculares com vista à formação das estruturas congeladas da semente de feijão.

Tem-se que a energia de ativação da cinética de congelamento do feijão é, respectivamente, de 3,95 e 2,85 $\mathrm{kJ} \mathrm{mol}^{-1}$, correspondente ao Modelo de Fourier utilizando o primeiro termo da série e o Modelo I de Cavalcanti-Mata \& Duarte. Os valores estão inferiores aos obtidos por Amaral et al. (2010) para as sementes de grão de bico congeladas de -23 a $-196{ }^{\circ} \mathrm{C}$, em que a energia de ativação foi de $18,78 \mathrm{~kJ} \mathrm{~mol}^{-1}$ para a semente com teor de água de $16 \%$ base úmida e de $16,36 \mathrm{~kJ} \mathrm{~mol}^{-1}$, para as sementes com teor de água de $20 \%$ base úmida.

A energia de ativação da reação corresponde à energia necessária para que a reação efetive menos a energia dos reagentes. Quanto mais baixa for a energia de ativação de uma reação mais elevada será sua velocidade (Bodner et al., 2011). Desta forma, a menor energia de ativação encontrada nas sementes de feijão implica em dizer que tal semente apresenta uma velocidade maior de congelamento.
A.

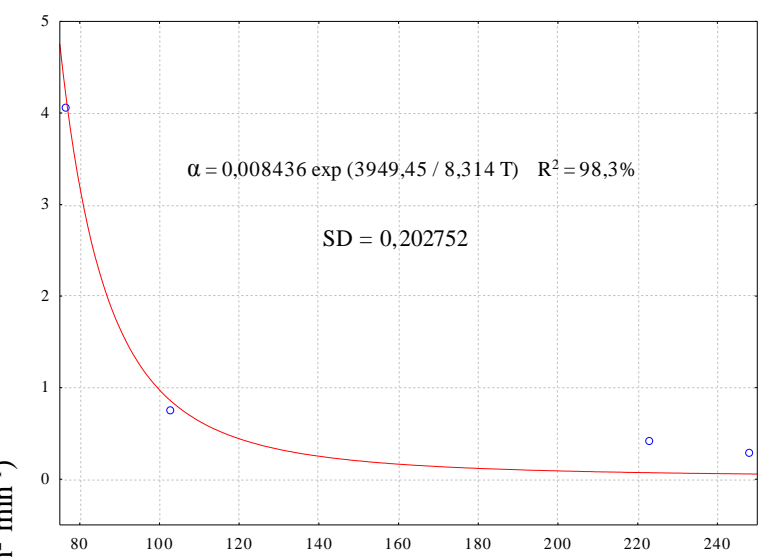

B.

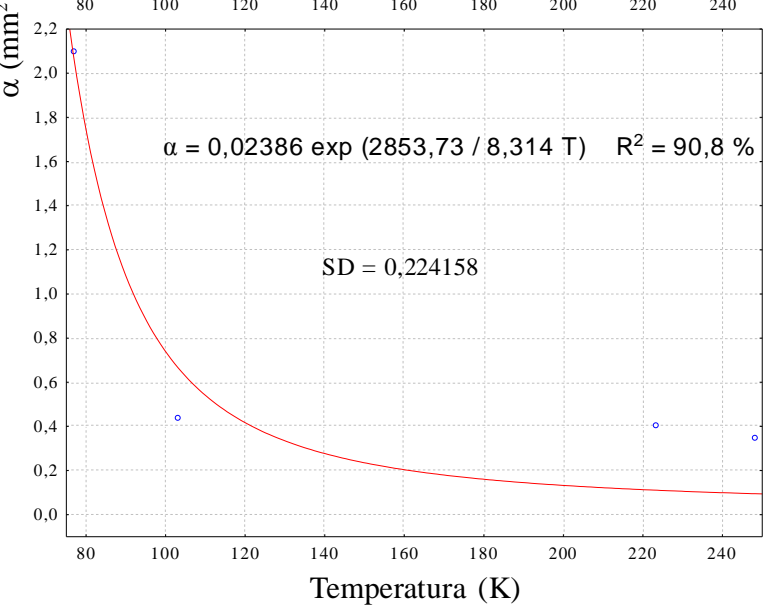

Figura 6. Difusividade térmica efetiva da semente de feijao em função da temperatura obtida do Modelo de Fourier (A) e do Modelo I de Cavalcanti-Mata \& Duarte (B) 


\section{Conclusões}

1. O Modelo de Fourier utilizando o $1^{\circ}$ termo da série, o Modelo I e Modelo II de Cavalcanti-Mata \& Duarte descrevem, de forma satisfatória, o processo de cinética de congelamento para o feijão cultivar 'gordo' nas temperaturas de -25 a $-196^{\circ} \mathrm{C}$.

2. Quanto maior o gradiente térmico a que as sementes são expostas maiores também a velocidade de congelamento e a difusividade térmica, ou seja, a difusividade térmica efetiva do produto aumenta com o incremento do diferencial de temperatura entre a amostra e o meio a que é submetido;

3. Os modelos estabelecidos para as sementes de feijão resultam em uma difusividade térmica efetiva que variou entre 0,2688 a 4,0457 $\mathrm{mm}^{2} \mathrm{~min}^{-1}$, e em uma energia de ativação de 3,95 $\mathrm{kJ} \mathrm{mol}^{-1}$ para o modelo de Fourier e de $2,85 \mathrm{~kJ} \mathrm{~mol}^{-1}$ para o Modelo Modelo I de Cavalcanti-Mata \& Duarte.

\section{Agradecimentos}

Os autores agradecem ao CNPq e à Universidade Federal de Campina Grande, pelo auxílio financeiro concedido em prol da execução desta pesquisa.

\section{LITERATURA CITADA}

Almeida, F. de A. C.; Jerônimo, E. de S.; Alves, N. M. C.; Gomes, J. P.; Silva, A. S. Estudo de técnicas para o armazenamento de cinco oleaginosas em condições ambientais e criogênicas. Revista Brasileira de Produtos Agroindustriais, v.12, p.189202, 2010.

Amaral, D. S. do; Sartor, F. R.; Félix, P. H. D.; Cavalcanti-Mata, M. E. R. M.; Duarte, M. E. M.; Moura Neto, L. G. de. Determinação da difusividade e da energia de ativação para o grão de bico com base na cinética de congelamento. In: Congresso de Pesquisa e Inovação da Rede Norte Nordeste de Educação Tecnológica - CONNEPI, 5, 2010, Maceió. Anais... Maceió: IFAL, 2010. 8p.

Barbin, D. F.; Davila, L.S. M.; Silveira Júnior, V. Avaliação da estabilidade de soluções modelo (cmc-sacarose) em recongelamentos. Boletim do CEPPA, v.28, p.125-132, 2010.

Becker, B. R.; Fricke, B. A. Food thermophysical property models. Heat and Mass Transfer, v.26, p.627-636, 1999.
Bodner, G. M.; Nakhleh, M. B.; Robinson, W. R. Activation energy. The chemical education web site. Division of Chemical Education at Purdue University, West Lafayette, IN, USA. In: http://w3.ufsm.br/juca/activate.htm. 12 Abr. 2011.

Bonomo, R. C. F.; Fontan, R. da C. I.; Souza, T. S. de; Veloso, C. M.; Reis, M. F. T.; Castro, S. de S. Thermophysical properties of cashew juice at different concentrations and temperatures. Revista Brasileira de Produtos Agroindustriais, v.11, p.35-42, 2009.

Cavalcanti-Mata, M. E. R. M. Tecnologia de crioconservação de sementes de urucum. Tecnologia \& Ciência Agropecuária, v.2, p.1-9, 2008.

Cavalcanti-Mata, M. E. R. M.; Duarte, M. E. M. Teoria e modelos matemáticos para congelamento de sementes. Revista Brasileira de Produtos Agroindustriais, v.13, p. 256-266, 2011.

Fikiin, K. A.; Fikiin, A. G. Predictive equations for thermophysical properties and enthalpy during cooling and freezing of food materials. Journal Food Engineering, v.40, p.1-6, 1999.

Goldfarb, M.; Duarte, M. E. M.; Cavalcanti-Mata, M. E. R. M.; Pimentel, L. W.; Severino, L. S. Cinética de congelamento criogênico de sementes de pinhão manso (Jatropha curcas L.). Engenharia Ambiental, v.7, p. 195-203, 2010.

Horn, M. M.; Martins, V. C. A.; Plepis, A. M. de G. Determinação da energia de ativação em hidrogéis poliméricos a partir de dados termogravimétricos. Polímeros, v.20, p.201-204, 2010.

IBGE - Instituto Brasileiro de Geografia e Estatística. Estatística da produção agrícola, 2011. 81p.

Mohapatra, D.; Rao, P. S. A thin layer drying model of parboiled wheat. Journal of Food Engineering, v.66, p.513-18, 2005.

Mohsenin, N. N. Thermal properties of foods and agricultural materials. New York: Gordon and Breach, Science Publishers, 1992. 407p.

Silva, W. P. da; Cavalcanti-Mata, M. E. R. M.; Silva, C. D. P. S. e; Guedes, M. A.; Lima, A. G. B. de. Determinação da difusividade e da energia de ativação para feijão macassar (Vigna unguiculata (L) Walp), variedade sempre verde, com base no comportamento da secagem. Engenharia Agrícola, v.28, p.325-333, 2008.

Tresena, N. de L.; Cavalcanti-Mata, M. E. R. M.; Duarte, M. E. M.; Moraes, A. M. de ; Dias, V. S. Qualidade fisiológica da semente de ipê rosa (Tabebuia heptahylla (Vellozo) Toledo) submetidas à crioconservação. Revista Brasileira de Produtos Agroindustriais, v.11, p.87-93, 2009. 